Flip Klijn

\title{
Constrained Allocation of Projects to Heterogeneous Workers with Preferences over Peers
}

\author{
${ }^{1}$ Institute for Economic Analysis (CSIC) and Barcelona CSE, Campus UAB, 08193 Bellaterra (Barcelona), Spain., E-mail: \\ flip.klijn@iae.csic.es. http://orcid.org/0000-0001-7255-6954.
}

\begin{abstract}
:
We study the problem of allocating projects to heterogeneous workers. The simultaneous execution of multiple projects imposes constraints across project teams. Each worker has preferences over the combinations of projects in which he can potentially participate and his team members in any of these projects. We propose a revelation mechanism that is Pareto-efficient and group strategy-proof (Theorem 1). We also identify two preference domains on which the mechanism is strongly group strategy-proof (Theorem 2). Our results subsume results by Monte and Tumennasan (2013) and Kamiyama (2013) .
\end{abstract}

Keywords: matching, allocation, heterogeneous agents, preferences over peers, efficiency, (group) strategyproofness

JEL classification: C78, D61, D78, I20

DOI: $10.1515 /$ bejte-2017-0038

\section{Introduction}

When a firm seeks for a smooth execution of a project it often has to ensure that the project's team has the right combination of skills. For instance, a cross-functional project may require a project manager, a certain number of analysts, technical personnel, and administrative staff. A particular example is that where a project has a minimal quorum (so that the project can be completed) and a maximum capacity (so that the firm does not run into inefficiencies). Moreover, when a firm is interested in carrying out multiple projects simultaneously, some workers might be required or are allowed to participate in more than one project. Hence, there can be constraints across teams that are to carry out distinct projects.

We study a general framework in which a firm has to allocate projects to workers. We assume that technical or physical constraints or any other requirements do not completely pin down the teams that are to execute (a subset of) the projects. Hence, there is room to take into account any possible preferences of the workers over the (sets of) projects in which they may be called upon to participate, and their possible co-workers. Our aim is to find a systematic and meaningful way of using the worker's preferences to obtain an allocation. More specifically, we are interested in finding Pareto-efficient allocations through a revelation mechanism. Since workers have to reveal their preferences, Pareto-efficiency with respect to the true preferences is only guaranteed if the mechanism satisfies some notion of strategy-proofness. We propose a revelation mechanism, called the serial shrink project allocation (SSPA) mechanism, that is Pareto-efficient and group strategy-proof (Theorem 1). ${ }^{1}$ The SSPA mechanism is based on a serial dictatorship in which, following a given hierarchy within the firm, each worker shrinks the set of feasible allocations by selecting those in which he is to participate in his (weakly) most preferred combinations of projects and co-workers. We show that group strategy-proofness cannot be strengthened to strong group strategy-proofness ${ }^{2}$ on the general domain (Example 1 and Example 2). However, for two interesting preference domains, strong group strategy-proofness can be established (Theorem 2).

Special cases of project allocation are considered in Monte and Tumennasan (2013) and Kamiyama (2013). Monte and Tumennasan (2013) study the situation in which (a) each worker can participate in at most one project, (b) workers only have preferences over projects (i.e., not over possible co-workers), (c) preferences are strict, and (d) each project has a minimum quorum and a maximum capacity (in particular, workers are homogeneous). Kamiyama (2013) considers more general allocation problems by relaxing Monte and Tumennasan (2013) assumption (d), i.e., constraints can be more complex. Here, we further generalize Kamiyama's framework by dropping assumptions (a)-(c). First, we allow for the possibility that a worker participates in multiple 
projects. Second, workers may not only have preferences over the projects in which they can become involved but over co-workers as well. Third, for some (but not all) of our results we do not require preferences to be strict.

Monte and Tumennasan (2013) and Kamiyama (2013) propose a revelation mechanism, called the (generalized) serial dictatorship with project closures ((G)SDPC) mechanism. Their main results show that the (G)SDPC mechanism is Pareto-efficient and strategy-proof. We show that in their frameworks our SSPA mechanism boils down to the (G)SDPC mechanism (Proposition 1 and Proposition 2). As a consequence, we obtain their results as corollaries and find that the $(\mathrm{G})$ SDPC mechanism is in fact strongly group strategy-proof (Corollary 1 and Corollary 2).

Apart from strengthening and generalizing existing results, our approach has other advantages. In the algorithm on which the (G)SDPC mechanism is based, an allocation is built up step by step. More precisely, at each step $i$, worker $i$ receives his most preferred assignment among those that are still "allowed," i.e., it is checked whether at future steps assignments can be arranged so that there is a feasible allocation where all workers $1, \ldots, i$ receive their chosen assignments. In the algorithm for the SSPA mechanism we take an equivalent yet more transparent approach. Initially, we consider all feasible allocations. Then, at each step $i$, worker $i$ further shrinks the set of (remaining) feasible allocations by selecting only those in which he receives his most preferred assignment. Therefore, in contrast with the (G)SDPC algorithm, the feasibility of the final allocation of the SSPA algorithm is immediate, i.e., does not require any proof. Finally, another advantage of our general framework is that the proofs of Pareto-efficiency and strategy-proofness become substantially more transparent since they do not contain specific arguments that hinge on quorums/capacities ( Monte and Tumennasan 2013) or graph theoretical notions ( Kamiyama 2013).

Early contributions of, among others, Barberà (1979), Dasgupta, Hammond, and Maskin (1979), and Pattanaik (1978) already study the immunity or vulnerability of mechanisms to joint manipulation of coalitions of agents (rather than individual agents) in different settings. More recently, Barberà, Berga, and Moreno (2010, 2016) study conditions under which strategy-proofness and group strategy-proofness are equivalent. More specifically, Barberà, Berga, and Moreno (2010) obtain sufficient and essentially necessary conditions on domains in public good economies. Barberà, Berga, and Moreno (2016) show that the equivalence between the two forms of strategy-proofness in many private good economies is due to a common structure. Even though project allocation is in general neither an instance of a pure public good economy nor pure private good economy, the two forms of strategy-proofness of our SSPA mechanism follow from the same arguments as is clear from the proof of Theorem 1 .

The remainder of the paper is organized as follows. In Section 2, we introduce the model. In Section 3, we present and prove the results. Section 4 concludes.

\section{Model}

A firm aims to allocate projects from a set $P=\left\{p_{1}, \ldots, p_{m}\right\}$ to a set of workers $I=\{1, \ldots, n\}$. Each project may have restrictions or requirements regarding the team of workers that can carry it out. Additionally, teams that are to execute different projects may have common workers, but possibly only to some extent. Taking into account all constraints, let the (feasible) project allocations be given by a non-empty set $\mathcal{A}$ with

$$
\mathcal{A} \subseteq\left\{\left\{\left(p^{1}, T^{1}\right), \ldots,\left(p^{k}, T^{k}\right)\right\}: \text { for all } l, l^{\prime}, l \neq l^{\prime}, \varnothing \neq T^{l} \subseteq I, p^{l} \in P \text {, and } p^{l} \neq p^{l^{\prime}}\right\}
$$

In other words, projects $p^{1}, \ldots, p^{k}$ can be simultaneously carried out by teams $T^{1}, \ldots, T^{k}$ if and only if $\left\{\left(p^{1}, T^{1}\right), \ldots,\left(p^{k}, T^{k}\right)\right\} \in A$.

Let $\alpha \in A$ be an allocation. Worker $i^{\prime}$ s assignment at $\alpha$ is given by

$$
\boldsymbol{\alpha}(\boldsymbol{i})=\bigcup_{\left(p^{l}, T^{l}\right) \in \alpha: i \in T^{l}}\left\{\left(p^{l}, T^{l}\right)\right\}
$$

Let $A(\mathbf{i})=\{\alpha(i): \alpha \in A\} \cup\{\varnothing\}$ denote worker $i^{\prime}$ s possible assignments, where $\emptyset$ is the empty assignment, i.e., being unassigned. We assume that worker $i$ has a weak order $\geq_{i}$ over $A(i)$. For $A, A^{\prime} \in A(i)$, we write $A>_{i} A^{\prime}$ if $A \geq_{i} A^{\prime}$ but not $A^{\prime}>_{i} A$, and we write $A \sim_{i} A^{\prime}$ if $A \geq_{i} A^{\prime}$ and $A^{\prime} \geq_{i} A$. For $A=\left\{\left(p^{1}, T^{1}\right), \ldots,\left(p^{k}, T^{k}\right)\right\} \in A(i)$, let $\boldsymbol{P}(\boldsymbol{A})=\left\{p^{1}, \ldots, p^{k}\right\}$ denote the projects associated with assignment $A$. Throughout, we assume that for each $A \in \mathcal{A}(i), A \geq_{i} \varnothing$. This assumption says that each non-empty assignment is considered a "good" which for instance delivers a bonus pay, promotion, or experience. Let $\mathcal{R}_{i}$ denote the collection of all possible preferences 
for $i$. Let $\mathcal{R}=\times_{i \in I} \mathcal{R}_{i}$. Since we do not change the set of projects, workers, and constraints, a (project allocation) problem is given by a preference profile $\geq=\left(\geq_{i}\right)_{i \in I} \in \mathcal{R}$.

Let $\alpha, \alpha^{\prime} \in \mathcal{A}$. Let $\geq \in \mathcal{R}$. Allocation $\alpha^{\prime}$ Pareto-dominates allocation $\alpha$ at $\geq$ if for each $i \in I, \alpha^{\prime}(i) \geq_{i} \alpha(i)$ and for some $j \in I, \alpha^{\prime}(j)>_{j} \alpha(j)$. Allocation $\alpha$ is Pareto-efficient at $\geq$ if there is no allocation $\alpha^{\prime}$ that Pareto-dominates $\alpha$ at $\geq$.

Let $\overline{\mathcal{R}}_{i} \subseteq \mathcal{R}_{i}$ and $\overline{\mathcal{R}}=x_{i \in I} \overline{\mathcal{R}}_{i}$. A mechanism on $\overline{\mathcal{R}}$ is a mapping $\varphi$ that associates each problem $\geq \in \overline{\mathcal{R}}$ with an allocation $\varphi(\geq) \in \mathcal{A}$. A mechanism $\varphi$ is Pareto-efficient if for each $\geq \in \overline{\mathcal{R}}, \varphi(\geq)$ is Pareto-efficient at $\geq$. A mechanism $\varphi$ is strategy-proof if there are no $i \in I, \geq \in \bar{R}$, and $\geq_{i}^{\prime} \in \bar{R}_{i}$ such that $\varphi_{i}\left(\geq_{i}^{\prime}\left(\geq_{j}\right)_{j \neq i}\right)>_{i} \varphi_{i}(\geq)$. A mechanism $\varphi$ is group strategy-proof if there are no $I^{\prime} \subseteq I, \geq \in \overline{\mathcal{R}}$, and $\left(\geq_{i}^{\prime}\right)_{i \in I^{\prime}} \in \times_{i \in I^{\prime}} \overline{\mathcal{R}}_{i}$ such that

$$
\text { for each } i \in I^{\prime}, \varphi_{i}\left(\left(\geq_{j}^{\prime}\right)_{j \in I^{\prime}}\left(\geq_{j}\right)_{j \notin I^{\prime}}\right)>_{i} \varphi_{i}(\geq) \text {. }
$$

A mechanism $\varphi$ is strongly group strategy-proof if there are no $I^{\prime} \subseteq I, \geq \in \overline{\mathcal{R}}$, and $\left(\geq_{i}^{\prime}\right)_{i \in I^{\prime}} \in \times_{i \in I^{\prime}} \overline{\mathcal{R}}_{i}$ such that

$$
\begin{aligned}
& \text { for each } i \in I^{\prime}, \varphi_{i}\left(\left(\geq_{j}^{\prime}\right)_{j \in I^{\prime}}\left(\geq_{j}\right)_{j \notin I^{\prime}}\right) \geq_{i} \varphi_{i}(\geq) \text { and } \\
& \text { for some } i^{\prime} \in I^{\prime}, \varphi_{i^{\prime}}\left(\left(\geq_{j}^{\prime}\right)_{j \in I^{\prime}}\left(\geq_{j}\right)_{j \notin I^{\prime}}\right)>_{i^{\prime}} \varphi_{i^{\prime}}(\geq) .
\end{aligned}
$$

Note that strong group strategy-proofness implies group strategy-proofness, which in turn implies strategyproofness.

\section{Results}

We propose a mechanism based on an algorithm that sequentially shrinks the set of project allocations to a singleton. The algorithm uses an order of the workers that can be thought of as going down through a hierarchy or an order of decreasing seniority. At each step of the algorithm a worker discards from the set of still available allocations all allocations that do not give him one of his most preferred assignments. Without loss of generality, throughout we order the workers according to their index: $1, \ldots, n$.

Shrink algorithm

Input: $\geq \in \mathcal{R}$.

- Step 0 (initialization): set $\Sigma_{0} \equiv \mathcal{A}$.

- Step $i=1, \ldots, n$ : let $\Sigma_{i} \equiv\left\{\alpha \in \Sigma_{i-1}: \alpha(i) \geq_{i} \alpha^{\prime}(i)\right.$ for each $\left.\alpha^{\prime} \in \Sigma_{i-1}\right\}$.

Output: $\Sigma(\geq) \equiv \Sigma_{n}$.

Obviously, since $A \neq \varnothing$ and all workers' preferences are complete, the algorithm is well-defined and the output contains at least one allocation. In general, the output is not a singleton, ${ }^{4}$ but according to the next lemma for each worker all allocations in the output report the same welfare.

\section{Lemma 1}

For each $\geq \in \mathcal{R}, \Sigma(\geq)$ only contains Pareto-efficient allocations. All allocations $\alpha, \alpha^{\prime} \in \Sigma(\geq)$ are Pareto equivalent at $\geq$, i.e., for each $i \in I, \alpha(i) \sim_{i} \alpha^{\prime}(i)$.

\section{Proof}

Suppose that for some $\geq \in \mathcal{R}$ an allocation $\alpha \in \Sigma(\geq)$. is Pareto-dominated by an allocation $\beta \in A$. Let $l \in I$ be the lowest index worker with $\beta(l)>_{l} \alpha(l)$. Since for each $j=1, \ldots, l-1, \beta(j) \sim_{j} \alpha(j)$, it follows that $\alpha, \beta \in \Sigma_{l-1}$. Then, since $\beta(l)>_{l} \alpha(l), \alpha \notin \Sigma_{l}$, which contradicts $\alpha \in \Sigma(\geq)$. The second statement follows from similar arguments.

In view of Lemma 1 , the firm can fix a tie-breaking rule so that for each $\geq \in \mathcal{R}$ we obtain a unique allocation $\sigma(\geq) \in \Sigma(\geq)$. The serial shrink project allocation (SSPA) mechanism $\sigma$ associated with the tie-breaking rule is the mechanism that for each $\geq \in \mathcal{R}$ yields the allocation $\sigma(\geq)$. If for some tie-breaking rule the associated SSPA mechanism is Pareto-efficient or ((strongly) group) strategy-proof on some preference domain, then all other SSPA mechanisms (associated with different tie-breaking rules) also are.

\section{Theorem 1}

SSPA mechanisms are Pareto-efficient and group strategy-proof on $\mathcal{R}$. 
proof

Pareto-efficiency follows from Lemma 1. Suppose $\sigma$ is not group strategy-proof, i.e., there are $I^{\prime} \subseteq I, \geq \in \mathcal{R}$, and $\left(\geq_{i}^{\prime}\right)_{i \in I^{\prime}} \in \times_{i \in I^{\prime}} \mathcal{R}_{i}$ such that

$$
\text { for each } i \in I^{\prime}, \sigma_{i}\left(\geq^{\prime}\right)>_{i} \sigma_{i}(\geq) \text {, where } \geq^{\prime}=\left(\left(\geq_{j}^{\prime}\right)_{j \in I^{\prime}}\left(\geq_{j}\right)_{j \notin I^{\prime}}\right) \text {. }
$$

Let $l$ be the lowest index worker in $I^{\prime}$. Then, from the fact that $A$ does not depend on the preference profile, it follows that steps $0, \ldots, l-1$ of the shrink algorithm for $\geq$ and $\geq^{\prime}$ are identical. So, at the beginning of step $l$ in the algorithm for $\geq$, there is an allocation (in $\Sigma_{l-1}$ ) where worker $l^{\prime}$ s assignment is $\sigma_{l}(\geq)$ and there is an allocation (in $\Sigma_{l-1}$ ) where worker $l^{\prime}$ s assignment is $\sigma_{l}\left(\succeq^{\prime}\right)$. From worker $l^{\prime}$ s optimization at step $l$ in the algorithm for $\succeq$, $\sigma_{l}(\geq) \geq_{l} \sigma_{l}\left(\geq^{\prime}\right)$, which contradicts eq. (1). Hence, $\sigma$ is group strategy-proof.

The following example shows that group strategy-proofness in Theorem 1 cannot be replaced by strong group strategy-proofness.

\section{Example 1}

(No SSPA mechanism is strongly group strategy-proof (I).)

Consider the problem with $P=\{p\}, I=\{1,2,3\}$, and $A=\{(p, 12),(p, 13)\},{ }^{5}$ i.e., the unique project can only be completed by either 12 or 13 . Let preferences $\geq \in \mathcal{R}$ be given by

$$
\begin{aligned}
& \geq_{1}:[(p, 12),(p, 13)], \emptyset \\
& \geq_{2}:(p, 12), \varnothing \\
& \geq_{3}:(p, 13), \varnothing
\end{aligned}
$$

The assignments are ranked from most preferred (left) to least preferred (right). The brackets in worker 1's preferences indicate that he is indifferent between his two non-empty assignments. Since $\Sigma(\geq)=(p, 12)$, at $\geq$ any SSPA mechanism yields the allocation $\sigma(\succeq)=(p, 12)$ in which the project is assigned to workers 1 and 2 (i.e., worker 3 is unassigned).

Let $I^{\prime}=\{1,3\}$ and suppose that worker 3 submits $\geq_{3}^{\prime}=\geq_{3}$ and worker 1 submits $\geq_{1}^{\prime} \in \mathcal{R}_{1}$ where

$$
\geq_{1}^{\prime}:(p, 13),(p, 12), \varnothing
$$

For $\geq^{\prime}=\left(\geq_{1}^{\prime}, \geq_{2}, \geq_{3}^{\prime}\right), \Sigma\left(\geq^{\prime}\right)=(p, 13)$. Hence, at $\geq^{\prime}$ any SSPA mechanism yields the allocation $\sigma\left(\geq^{\prime}\right)=(p, 13)$ in which the project is assigned to workers 1 and 3 . Clearly, $\sigma_{1}\left(\geq^{\prime}\right) \sim_{1} \sigma_{1}(\geq)$ and $\sigma_{3}\left(\geq^{\prime}\right) \succ_{3} \sigma_{3}(\succeq)$. Hence, no SSPA mechanism is strongly group strategy-proof. $\diamond$

A key feature of Example 1 is that a high hierarchy worker (worker 1) who is indifferent between teams with the same project can "help" a lower hierarchy worker (worker 3) to be assigned to the project (or even to a different, more preferred project) by pretending that he (i.e., worker 1) strictly prefers one team over the other(s).

To avoid a manipulation as in Example 1, one possibility is to assume that for each $i \in I$, worker $i^{\prime}$ s preferences $\geq_{i}$ are strict, i.e., for all $A, A^{\prime} \in A(i)$ with $A \neq A^{\prime}, A \varkappa_{i} A^{\prime}$. Let $\mathcal{R}_{i}^{>} \subseteq \mathcal{R}_{i}$ denote the collection of all possible strict preferences for $i$. Let $\mathcal{R}^{>}=\times_{i \in I} \mathcal{R}_{i}^{>}$. According to Theorem 2 below, if preferences are strict, the unique SSPA mechanism on $\mathcal{R}^{>}$is strongly group strategy-proof.

A second possible idea to avoid a manipulation as in Example 1 is to assume that for each $i \in I$, worker $i^{\prime}$ s preferences $\geq_{i}$ are indifferent with respect to peers, i.e., for all $A, A^{\prime} \in A(i), A \sim_{i} A^{\prime}$ if $P(A)=P\left(A^{\prime}\right)$. This assumption ensures that no worker submits a preference list in which a project with some co-worker is strictly preferred to the same project with another co-worker (such as in $\geq_{1}^{\prime}$ in Example 1). However, as the following example shows, this assumption is not sufficient to guarantee that SSPA mechanisms are strongly group strategy-proof.

\section{Example 2}

(No SSPA mechanism is strongly group strategy-proof (II).)

Consider the problem with $P=\{p\}, I=\{1,2,3\}$, and $\mathcal{A}=\left\{\left(p_{1}, 123\right),\left(p_{2}, 12\right)\right\}$. Let preferences $\geq \in \mathcal{R}$ be given by

$$
\begin{aligned}
& \geq_{1}:\left[\left(p_{1}, 123\right),\left(p_{2}, 12\right)\right], \varnothing \\
& \geq_{2}:\left(p_{2}, 12\right),\left(p_{1}, 123\right), \varnothing \\
& \geq_{3}:\left(p_{1}, 123\right), \varnothing
\end{aligned}
$$

Note that since each project can only be completed by one team, all preferences are (trivally) indifferent with respect to peers. Since $\Sigma(\geq)=\left(p_{2}, 12\right)$, at $\geq$ all SSPA mechanisms yield the allocation $\sigma(\geq)=\left(p_{2}, 12\right)$. Let $I^{\prime}=\{1,3\}$ and suppose that worker 3 submits $\geq_{3}^{\prime}=\geq_{3}$ and worker 1 submits $\geq_{1}^{\prime} \in \mathcal{R}_{1}$ where

$$
\geq_{1}^{\prime}:\left(p_{1}, 123\right),\left(p_{2}, 12\right), \varnothing
$$


Let $\geq^{\prime}=\left(\geq_{1}^{\prime}, \geq_{2}, \geq_{3}^{\prime}\right)$. Since $\Sigma\left(\geq^{\prime}\right)=\left(p_{1}, 123\right)$, at $\geq^{\prime}$ all SSPA mechanisms yield the allocation $\sigma\left(\geq^{\prime}\right)=\left(p_{1}, 123\right)$. Clearly, $\sigma_{1}\left(\geq^{\prime}\right) \sim_{1} \sigma_{1}(\geq)$ and $\sigma_{3}\left(\geq^{\prime}\right)>_{3} \sigma_{3}(\geq)$. Hence, no SSPA mechanism is strongly group strategy-proof. $\diamond$

We strengthen the previous assumption by demanding that for each $i \in I$, worker $i^{\prime}$ s preferences $\geq_{i}$ are indifferent with respect to peers only, i.e., for all $A, A^{\prime} \in \mathcal{A}(i), A \sim_{i} A^{\prime}$ if and only if $P(A)=P\left(A^{\prime}\right)$. This assumption excludes a worker from being indifferent between two assignments that involve different projects (such as in $\geq_{1}$ in Example 2). More precisely, if $\geq_{i}$ is indifferent with respect to peers only, then agent $i^{\prime}$ s preferences are purely based on the (combinations of) projects (and not on the teammates), and moreover, preferences over (combinations of) projects are strict. Let $\mathcal{R}_{i}^{=} \subseteq \mathcal{R}_{i}$ denote the collection of all possible preferences for $i$ where he is indifferent with respect to peers only. Let $\mathcal{R}^{=}=\times_{i \in I} \mathcal{R}_{i}^{=}$. Domains $\mathcal{R}^{>}$and $\mathcal{R}^{=}$have natural appeal. Still, these domains are of course somewhat restricted if we believe that in practical applications agents have (more complicated) indifferences in their preferences. We can now state and prove our second main result.

\section{Theorem 2} proof.

Let $\bar{R}=R^{>}, \mathcal{R}^{=}$. Then, there is a unique SSPA mechanism on $\overline{\mathcal{R}}$ which moreover is strongly group strategy-

Proof

Let $\bar{R}=\mathcal{R}^{>}, \mathcal{R}^{=}$. Suppose that there are distinct $\alpha, \alpha^{\prime} \in A, \alpha \neq \alpha^{\prime}$, contained in the output of the shrink algorithm for some $\geq \in \mathcal{R}$. Let $i \in I$ such that $P(\alpha(i)) \neq P\left(\alpha^{\prime}(i)\right)$. Since $\geq_{i} \in \overline{\mathcal{R}}_{i}, \alpha(i) \sim_{i} \alpha^{\prime}(i)$, which contradicts the optimization at step $i$ of the shrink algorithm. So, for each $\geq \in \overline{\mathcal{R}},|\Sigma(\geq)|=1$. So, there is a unique SSPA mechanism $\sigma$ on $\overline{\mathcal{R}}$.

Suppose $\sigma$ is not strongly group strategy-proof on $\overline{\mathcal{R}}$. Then, there are $I^{\prime} \subseteq I, \geq \in \overline{\mathcal{R}}$, and $\left(\geq_{i}^{\prime}\right)_{i \in I^{\prime}} \in \times_{i \in I^{\prime}} \overline{\mathcal{R}}_{i}$ such that

$$
\begin{aligned}
& \text { for each } i \in I^{\prime}, \sigma_{i}\left(\geq^{\prime}\right) \geq_{i} \sigma_{i}(\geq) \text { and } \\
& \text { for some } i^{\prime} \in I^{\prime}, \sigma_{i^{\prime}}\left(\geq^{\prime}\right)>_{i^{\prime}} \sigma_{i^{\prime}}(\geq),
\end{aligned}
$$

where $\geq^{\prime}=\left(\left(\geq_{j}^{\prime}\right)_{j \in I^{\prime}},\left(\geq_{j}\right)_{j \notin I^{\prime}}\right)$. Let $l$ be the lowest index worker in $I^{\prime}$ for which $\sigma_{l}\left(\geq^{\prime}\right)>_{l} \sigma_{l}(\succeq)$. Let $\Sigma_{0}, \Sigma_{1}, \ldots, \Sigma_{n}$ and $\Sigma_{0}^{\prime}, \Sigma_{1}^{\prime}, \ldots, \Sigma_{n}^{\prime}$ be the sets of allocations in the algorithm for $\geq$ and $\geq^{\prime}$. Obviously, $\Sigma_{0}=\Sigma_{0}^{\prime}$. Suppose that for some $k$ with $0 \leq k<l-1$ we have $\Sigma_{k}=\Sigma_{k}^{\prime}$. We prove that $\Sigma_{k+1}=\Sigma_{k+1}^{\prime}$. The statement follows immediately if $k+1 \notin I^{\prime}$. Suppose $k+1 \in I^{\prime}$. Since $k+1<l, \sigma_{k+1}\left(\geq^{\prime}\right) \sim_{k+1} \sigma_{k+1}(\geq)$. Hence, worker $k+1$ shrinks the set of allocations at $\geq$ and $\geq^{\prime}$ in exactly the same way. (Here we use that $\geq_{k+1}^{\prime} \in \bar{R}_{k+1}$.) Hence, $\Sigma_{k+1}=\Sigma_{k+1}^{\prime}$. We conclude that $\Sigma_{l-1}=\Sigma_{l-1}^{\prime}$. But then $\sigma_{l}\left(\geq^{\prime}\right)>_{l} \sigma_{l}(\geq)$ contradicts worker l's optimization at step $l$ of the algorithm for $\geq$. Hence, $\sigma$ is strongly group strategy-proof on $\bar{R}$.

Next, we discuss two classes of allocation problems that have been studied in the literature and derive two corollaries to Theorem 1 and Theorem 2.

\subsection{Two Classes of Allocation Problems}

Monte and Tumennasan (2013) study so-called matching with quorums. Their model is a special case of ours in two aspects. First, each project $p$ requires at least $q_{p} \geq 1$ workers (its minimum quorum) to be completed and has a maximum capacity $k_{p} \leq n$. Workers are assumed to be homogeneous. Hence, the feasible project allocations are given by $\mathcal{A}=\mathcal{A}^{q}$ where

$$
\begin{array}{r}
\mathcal{A}^{q}=\left\{\left\{\left(p^{1}, T^{1}\right), \ldots,\left(p^{k}, T^{k}\right)\right\}: \text { for all } l, l^{\prime}, l \neq l^{\prime}, T^{l} \subseteq I, p^{l} \in P, p^{l} \neq p^{l^{\prime}},\right. \\
\left.T^{l} \cap T^{l^{\prime}}=\varnothing, \text { and } q_{p^{l}} \leq\left|T^{l}\right| \leq k_{p^{l}}\right\} .
\end{array}
$$

In particular, each worker $i \in I$ can be assigned to at most one project $\left(T^{l} \cap T^{l^{\prime}}=\varnothing\right)$, i.e., for each assignment $A \in$ $\mathcal{A}(i),|A| \leq 1$. Second, workers are indifferent with respect to peers only. Monte and Tumennasan (2013) propose a mechanism (on $\mathcal{R}^{=}$) called the serial dictatorship with project closures (SDPC) mechanism. They show that the SDPC mechanism is Pareto-efficient and strategy-proof ( Monte and Tumennasan 2013, Theorem 1). In matching with quorums, the SDPC mechanism coincides with the unique SSPA mechanism (see Proposition 2, Appendix A). Hence, we obtain the following corollary to Theorem 1 and Theorem 2. 


\section{Corollary 1}

(Matching with quorums.)

The serial dictatorship with project closures (SDPC) mechanism in matching with quorums is Paretoefficient and strongly group strategy-proof.

Kamiyama (2013) studies so-called abstract matching markets. His model subsumes that of Monte and Tumennasan (2013), yet it is still a special case of ours in again two aspects. First, in Kamiyama (2013) there is a finite bipartite graph $G=(V, E)$ where $V=I \cup P$ is the set of vertices and $E$ is a (possibly incomplete) set of edges between vertices in $I$ and vertices in $P$. There is also a non-empty family $F_{e}$ of subsets of $E$ such that for each $F \in \mathcal{F},\left[\{i, p\},\left\{i, p^{\prime}\right\} \in F \Rightarrow p=p^{\prime}\right]$. The feasible project allocations are given by $\mathcal{A}=\mathcal{A}^{a}$ where

$$
\begin{array}{r}
\mathcal{A}^{a}=\left\{\left\{\left(p^{1}, T^{1}\right), \ldots,\left(p^{k}, T^{k}\right)\right\}: \text { for all } l, l^{\prime}, l \neq l^{\prime}, \varnothing \neq T^{l} \subseteq I, p^{l} \in P, p^{l} \neq p^{l^{\prime}},\right. \\
\text { and } \left.\bigcup_{l^{\prime \prime}} \bigcup_{i \in T^{l^{\prime \prime}}}\left\{\left\{i, p^{l^{\prime \prime}}\right\}\right\} \in \mathcal{F}\right\} .
\end{array}
$$

In particular, each worker can only be assigned to at most one project. Second, workers are indifferent with respect to peers only. Kamiyama (2013) proposes a mechanism (on $\mathcal{R}^{=}$) called the generalized serial dictatorship with project closures (GSDPC) mechanism. He shows that the GSDPC mechanism is Pareto-efficient and strategy-proof ( Kamiyama 2013, Theorem 2). In abstract matching markets, the GSDPC mechanism coincides with the unique SSPA mechanism (see Proposition 1, Appendix A). Hence, we obtain the following corollary to Theorem 1 and Theorem 2.

\section{Corollary 2}

(Abstract matching markets.)

The generalized serial dictatorship with project closures (GSDPC) mechanism in abstract matching markets is Pareto-efficient and strongly group strategy-proof.

\section{Concluding Remarks}

We have studied a general framework to allocate projects to workers. We have assumed that each worker submits a preference list where all his possible assignments are ranked weakly above the empty assignment. Even though this is restrictive, we can think of situations in which the firm can call upon its workers to participate in projects, e.g., because of conditions in employment contracts, and hence workers should be ready to do so. Especially when projects are "goods" (as is the case in our framework) the assumption seems very reasonable. Note that Kamiyama (2013) and Monte and Tumennasan (2013) make this assumption as well.

If we would like workers to have preferences and submit lists where some assignments could be declared "unacceptable," then the definitions related to mechanisms can be adjusted as follows. Let $\mathcal{R}_{i}^{*}$ denote the collection of weak orders ${ }^{6}$ over $\mathcal{A}(i)$. Let $\mathcal{R}^{*}=\times_{i \in I} \mathcal{R}_{i}^{*}$. A mechanism on $\mathcal{R}^{*}$ is a mapping $\varphi$ that associates each problem $\geq \in \mathcal{R}^{*}$ with an allocation $\varphi(\geq) \in \mathcal{A} \cup\{\varnothing\}$. Pareto-efficiency and strategy-proofness of mechanisms are defined as before. In the case of unacceptable assignments, it is natural to also include individual rationality as a desirable property of a mechanism. A mechanism $\varphi$ is individually rational if for each $\geq \in \mathcal{R}^{*}, \alpha=\varphi(\geq)$ is individually rational at $\geq$, i.e., for each $i \in I, \alpha(i) \geq_{i} \varnothing$.

The following example shows that there is no individually rational mechanism on $\mathcal{R}^{*}$ that is both Paretoefficient and strategy-proof.

\section{Example 3}

\section{(Unacceptable assignments.)}

Consider the problem with $P=\left\{p_{1}, p_{2}\right\}, I=\{1,2\}$, and $\mathcal{A}=\left\{\left(p_{1}, 12\right),\left(p_{2}, 12\right)\right\}$. Let $\varphi$ be an individually rational and Pareto-efficient mechanism on $\mathcal{R}^{*}$. Let preferences $\geq \in \mathcal{R}^{*}$ be given by

$$
\begin{aligned}
& \geq_{1}:\left(p_{1}, 12\right),\left(p_{2}, 12\right), \varnothing \\
& \geq_{2}:\left(p_{2}, 12\right),\left(p_{1}, 12\right), \varnothing .
\end{aligned}
$$

At $\geq$ there are two individually rational and Pareto-efficient allocations, namely both workers are assigned to either $p_{1}$ or $p_{2}$. Suppose mechanism $\varphi$ selects $p_{1}$. Then, consider

$$
\geq_{2}^{\prime}:\left(p_{2}, 12\right), \emptyset,\left(p_{1}, 12\right)
$$


i.e., worker 2 reports $p_{1}$ to be unacceptable. Obviously, at $\geq^{\prime}=\left(\geq_{1}, \geq_{2}^{\prime}\right) \in \mathcal{R}^{*}$, the unique individually rational and Pareto-efficient allocation assigns both workers to project $p_{2}$. Hence, at $\geq$ worker 2 can manipulate mechanism $\varphi$ and obtain a more preferred assignment. If the mechanism selects $p_{2}$ at $\geq$, then similarly worker 1 can manipulate the mechanism. Hence, there is no individually rational mechanism that is both Pareto-efficient and strategy-proof. $\diamond$

Finally, note that in general there are multiple Pareto-efficient allocations. Then, a natural question that comes to mind is whether each Pareto-efficient allocation can be obtained as the outcome of some SSPA mechanism (by possible changing the order of the workers). The example in Monte and Tumennasan (2013), p.17 shows that the answer is already negative for the specific case of matching with quorums.

\section{Acknowledgements}

I thank Ronald Peeters and a reviewer for useful comments and suggestions. I gratefully acknowledge financial support from the Generalitat de Catalunya (2014-SGR-1064), the Spanish Ministry of Economy and Competitiveness through Plan Nacional I+D+i (ECO2014-59302-P), and the Severo Ochoa Programme for Centres of Excellence in R \& D (SEV-2015-0563). A first draft was written while I was visiting the Aix-Marseille School of Economics. Their hospitality and financial support is gratefully acknowledged.

\section{Funding}

Agència de Gestió d'Ajuts Universitaris i de Recerca, (Grant / Award Number: '2014-SGR-1064') Aix-Marseille School of Economics, (Grant / Award Number: ) Spanish Ministry of Economy and Competitiveness, (Grant / Award Number: 'ECO2014-59302-P') Severo Ochoa Programme for Centres of Excellence in R\&D, (Grant / Award Number: 'SEV-2015-0563')

\section{Appendix}

\section{A Appendix}

Kamiyama (2013, Section 4.2) shows that the class of matching markets with quorums is a special class of abstract matching markets and that for matching with quorums the SDPC mechanism coincides with the GSDPC mechanism. Hence, it is sufficient to establish the equivalence of the GSDPC mechanism with the serial shrink project allocation mechanism for abstract matching markets. We first introduce an equivalent way to specify feasible project allocations.

Let $\bar{P}=P \cup\{\varnothing\}$. A matching is a correspondence $\mu: \bar{P} \rightarrow I$, i.e., for each $p \in \bar{P}, \mu(p) \subseteq I$, such that $\bigcup_{p \in \bar{P}} \mu(p)=I$ and for all $p, p^{\prime} \in \bar{P}$ with $p \neq p^{\prime}, \mu(p) \cap \mu\left(p^{\prime}\right)=\emptyset$. Let $\alpha \in \mathcal{A}$ be a feasible project allocation. Then, $\mu$ defined by

$$
\mu(p)= \begin{cases}T & \text { if } p \in P \text { and } T \subseteq I \text { is such that }(p, T) \in \alpha \\ \varnothing & \text { if } p \in P \text { and there is no } T \subseteq I \text { with }(p, T) \in \alpha\end{cases}
$$

and $\mu(\phi)=I \backslash \bigcup_{p \in P} \mu(p)$ is a matching. Matchings that are thus obtained from feasible project allocations are called feasible matchings.

\section{Lemma 2}

There is a one-to-one correspondence between feasible matchings and feasible project allocations.

\section{Proof}

The statement follows from the observation that if $\mu$ is a feasible matching, then

$$
\alpha=\bigcup_{p \in P: \mu(p) \neq \emptyset}\{(p, \mu(p))\}
$$

is the feasible project allocation that induces $\mu$. 
Let an abstract matching market (Kamiyama 2013) have feasible project allocations $A^{a}$, i.e., as specified in eq. (2). One easily verifies that a matching $v$ is feasible if and only if

$$
\bigcup_{\substack{p \in P: \\ \nu(p) \neq \varnothing}} \bigcup_{i \in v(p)}\{\{i, p\}\} \in \mathcal{F}
$$

In abstract matching markets, workers are indifferent with respect to peers only. So, all allocation problems are in $\mathcal{R}^{=}$. Then, for convenience, we can omit co-workers from the description of each worker's assignment (at any allocation). Similarly, with slight abuse of notation, we can use $\geq_{i}$ to denote worker $i^{\prime}$ s linear order over projects (and being unassigned). In particular, for all $p, p^{\prime} \in P$ with $p \neq p^{\prime}$ we will write $p \geq_{i} p^{\prime}$ if and only if for all/some $\{(p, T)\},\left\{\left(p^{\prime}, T^{\prime}\right)\right\} \in \mathcal{A}(i),\{(p, T)\} \geq_{i}\left\{\left(p^{\prime}, T^{\prime}\right)\right\}$. We similarly slightly abuse the notation $>_{i}$.

Fix the order of workers as $1, \ldots, n$. The GSDPC algorithm can be described as follows.

GSDPC algorithm ( Kamiyama 2013 )

Input: $\geq \in \mathcal{R}^{=}$.

- Step 0 (initialization): for each $p \in \bar{P}$, set $\mu(p) \equiv \emptyset$.

- Step $i=1, \ldots, n$ :

I. Compute

$$
\left.P_{i}=\left\{p \in P \text { : for some } F \in F_{F},[\{i, p\}\} \cup \bigcup_{\substack{p^{\prime} \in P: \\ \mu\left(p^{\prime}\right) \neq \varnothing}} \bigcup_{j \in \mu\left(p^{\prime}\right)}\left\{\left\{j, p^{\prime}\right\}\right\}\right] \subseteq F\right\} \cup\{\emptyset\} .
$$

II. Let $p^{*} \in P_{i}$ such that for each $p \in P_{i} \backslash\left\{p^{*}\right\}, p^{*}>_{i} p$.

Adjust $\mu\left(p^{*}\right) \equiv \mu\left(p^{*}\right) \cup\{i\}$.

Output: a feasible matching $\mu^{\geq} \equiv \mu$.

The GSDPC mechanism yields for each $\geq \in \mathcal{R}^{=}$the feasible matching $\mu^{\geq}$obtained by applying the GSDPC algorithm to $\geq$.

\section{Proposition 1}

In abstract matching markets, the GSDPC mechanism coincides with the unique SSPA mechanism. Formally, for each $\geq \in R^{=}$, each worker is assigned to the same project (or no project) at $\mu^{\geq}$and $\sigma(\geq)$.

\section{Proof}

Let $\geq \in \mathcal{R}^{=}$. From eq. (3), it follows that for each $i=1, \ldots, n$,

$P_{i}=\{p \in P$ : there is a feasible matching $v$ such that

$$
\left.\left[\{\{i, p\}\} \cup \bigcup_{\substack{p^{\prime} \in P: \\ \mu\left(p^{\prime}\right) \neq \varnothing}} \bigcup_{j \in \mu\left(p^{\prime}\right)}\left\{\left\{j, p^{\prime}\right\}\right\}\right] \subseteq \bigcup_{\substack{p^{\prime} \in P: \\ \nu\left(p^{\prime}\right) \neq \varnothing}} \bigcup_{j \in v\left(p^{\prime}\right)}\left\{\left\{j, p^{\prime}\right\}\right\}\right\} \cup\{\varnothing\} .
$$

Using Lemma 2 one can now easily prove by induction that for each $i=1, \ldots, n$, if $p^{*} \in P_{i}$ is such that $p^{*} \neq \varnothing$ and for each $p \in P_{i} \backslash\left\{p^{*}\right\}, p^{*}>_{i} p$, then for each $\alpha \in \Sigma_{i}$ in the shrink algorithm for $\geq, p^{*}$ is the unique project in $P(\alpha(i))$. Similarly, if for each $p \in P_{i} \backslash\{\varnothing\}, \emptyset>_{i} p$, then for each $\alpha \in \Sigma_{i}$ in the shrink algorithm for $\geq, P(\alpha(i))=\emptyset$. Hence, each worker is assigned to the same project (or no project) at $\mu^{\geq}$and $\sigma(\geq)$.

The following result is an immediate corollary to Proposition 1 and Kamiyama (2013, Section 4.2).

\section{Proposition 2}

In matching with quorums, the SDPC mechanism coincides with the unique SSPA mechanism.

\section{Notes}

${ }^{1} \mathrm{~A}$ mechanism is group strategy-proof if no group of workers can misrepresent their preferences in such a way that all its workers obtain a strictly more preferred assignment.

${ }^{2} \mathrm{~A}$ mechanism is strongly group strategy-proof if no group of workers can misrepresent their preferences in such a way that all its workers obtain a weakly more preferred assignment and at least one worker a strictly more preferred assignment.

${ }^{3}$ In other words, $\geq_{i}$ is complete and transitive.

${ }^{4}$ A simple example is $I=\{1\}, P=\left\{p_{1}, p_{2}\right\}, \mathcal{A}=\left\{\left\{\left(p_{1},\{1\}\right)\right\},\left\{\left(p_{2},\{1\}\right)\right\}\right\}$ and $\left\{\left(p_{1},\{1\}\right)\right\} \sim_{1}\left\{\left(p_{2},\{1\}\right)\right\}$. 
${ }^{5}$ For notational convenience and when no confusion can arise, we write $i_{1} i_{2} \ldots i_{k}$ for the set $\left\{i_{1}, i_{2}, \ldots, i_{k}\right\}$ and omit brackets from singleton sets. So, $A=\{\{(p,\{1,2\})\},\{(p,\{1,3\})\}\}$ is written as $A=\{(p, 12),(p, 13)\}$.

${ }^{6}$ So, since $\emptyset \in \mathcal{A}(i)$, we allow for preferences $\geq_{i} \in \mathcal{R}_{i}^{*}$ and $A \in \mathcal{A}(i)$ with $\emptyset>_{i} A$.

${ }^{7}$ Kamiyama (2013) requires in the definition of $P_{i}$ that $F \backslash\left[\{\{i, p\}\} \cup \underset{\substack{p^{\prime} \in P: \\ \mu\left(p^{\prime}\right) \neq \emptyset}}{\bigcup} \bigcup_{j \in \mu\left(p^{\prime}\right)}\left\{\left\{j, p^{\prime}\right\}\right\}\right]$ only contains edges that involve (a subset of) workers $i+1, \ldots, n$ (and any projects). However, this requirement is redundant by his assumptions that (1) for each $F \in \mathcal{F}_{,},\left[\{j, q\},\left\{j, q^{\prime}\right\} \in\right.$ $\left.F \Rightarrow q=q^{\prime}\right]$ and (2) for each $\{j, q\} \in E, q>_{j} \varnothing$.

\section{References}

Barberà, S. 1979. “A Note on Croup Strategy-Proof Decision Schemes." Econometrica 47: 637-640.

Barberà, S., D. Berga, and B. Moreno. 2010. "Individual versus Group Strategy-Proofness: When Do They Coincide?" Journal of Economic Theory 145 (5): 1648-1674.

Barberà, S., D. Berga, and B. Moreno. 2016. “Group Strategy-Proofness in Private Good Economies.” American Economic Review 106 (4): 1073-1099.

Dasgupta, P., P. Hammond, and E. Maskin. 1979. “The Implementation of Social Choice Rules: Some Ceneral Results on Incentive Compatibility." Review of Economic Studies 46 (2): 185-216.

Green, J., and ].-J. Laffont. 1979. “On Coalition Incentive Compatibility.” Review of Economic Studies 46 (2): 243-254.

Kamiyama, N. 2013. "A Note on the Serial Dictatorship with Project Closures." Operations Research Letters 41 (5): 559-561.

Monte, D., and N. Tumennasan. 2013. "Matching with Quorums." Economics Letters 120 (1): 14-17.

Pattanaik, P.K. 1978. Strategy and Croup Choice. Amsterdam: North-Holland Publishing Company. 